\title{
Lock-in thermography for characterization of nuclear materials
}

\author{
Alexandre Semerok ${ }^{*}$, Sang Pham Tu Quoc, Guy Cheymol, Catherine Gallou, Hicham Maskrot, and Gilles Moutiers \\ Den-Service d'Études Analytiques et de Réactivité des Surfaces (SEARS), CEA, Université Paris-Saclay, 91191 Gif-sur-Yvette, \\ France
}

Received: 23 September 2015 / Received in final form: 2 February 2016 / Accepted: 22 February 2016

Published online: 15 April 2016

\begin{abstract}
A simplified procedure of lock-in thermography was developed and applied for characterization of nuclear materials. The possibility of thickness and thermal diffusivity measurements with the accuracy better than $90 \%$ was demonstrated with different metals and Zircaloy-4 claddings.
\end{abstract}

\section{Introduction}

Lock-in thermography is a non-destructive method which may be applied to test and to ensure remote control over materials in severe environment (e.g. nuclear installations) in a wide temperature range. The method is based on the laser heating of a sample with a modulated laser power at a given frequency $f(\mathrm{~Hz})$ followed by measurements of a thermal radiation emitted by the sample. The phase shifts $\Delta \varphi$ between the laser power and the thermal radiation measured at different modulated frequencies are then compared with those obtained with an analytical $(3 \mathrm{D}+\mathrm{t})$ model developed at the LISL (DEN/DANS/DPC/SEARS) in case of the heating of a sample covered by a deposited layer [1,2]. Thus, it is possible to provide a tool to characterize some sample properties (thickness, thermal diffusivity, deposited layer/surface thermal contact resistance, characterization of under-surface defects and their evolution with time). The phase shift of heating temperature is presented in Figure 1.

\section{Model for the heating of a plate}

In a thermal model for homogeneous and isotropic plate with infinite dimensions, we supposed that:

- variations of the optical and thermal properties for a surface covered by a deposited layer during its heating are negligible;

- the surface roughness effect and heat exchange due to the sample surface/air convection are also negligible.

\footnotetext{
* e-mail: alexandre.semerok@cea.fr
}



Fig. 1. Phase shift of heating temperature.

In the heating models $[1,2]$ for a surface with a deposited layer, we supposed that the layer/surface thermal resistance $(\Re)$ and the layer optical thickness $(\alpha L)$ on laser wavelength are very high $(\Re \rightarrow \infty$ and $\alpha L>>1)$. By applying the Fourier series analysis to the intensity of the laser beam and the temperature in the stationary regime of the laser heating $[2,3]$, the complex temperature amplitude of the front face of a plate can be written as:

$$
\begin{aligned}
\Delta T(z, r)= & \frac{\alpha(1-R) \tilde{I}^{+\infty}}{k} \int_{0}^{+\infty} \frac{\Theta(\xi)}{\xi^{2}-\alpha^{2}-\frac{2 \pi i f C_{v}}{k}}\left(\left(\frac{\alpha e^{-\Psi L}}{\Psi\left(e^{\Psi L}-e^{-\Psi L}\right)}+\frac{\alpha}{\Psi}\right) e^{-\Psi z}\right. \\
& \left.+e^{-\Psi z}+\frac{\alpha e^{-\Psi L}}{\Psi\left(e^{\Psi L}-e^{-\Psi L}\right)} e^{\Psi z}-e^{-\alpha z}\right) J_{0}(\xi r) d \xi
\end{aligned}
$$

with $\Theta(\xi)=\frac{\xi r_{0}^{2}}{2} \exp \left(-\frac{\xi^{2} r_{0}^{2}}{4}\right)$ for the Gaussian beam; $\Psi=\sqrt{\xi^{2}-2 \pi i f C_{v} / k} ; \quad \tilde{I}=i \sqrt{a_{1}^{2}+b_{1}^{2}} e^{-i \Phi_{L P}} ;$ $\Phi_{L P}=\operatorname{atan}\left(\frac{b_{1}}{a_{1}}\right) ; \quad J_{0}(\xi r)=\frac{1}{\pi} \int_{0}^{\pi} \cos (-\xi r \sin \tau) d \tau$; $a_{1}=2 f \int_{0}^{1 / f} I(t) \cos (2 \pi f t) d t$;

$b_{1}=2 f \int_{0}^{1 / f} I(t) \sin (2 \pi f t) d t ; I(t)=I_{0}(1-\cos (2 \pi f t))$, 
where: $z$ and $r$, respectively, are the propagation direction of the laser beam and the radial distance from the center of the heated zone at the sample surface; $C_{\mathrm{v}}, k$ and $L$ : the volumetric specific heat, the thermal conductivity and the thickness of the sample; $\alpha$ and $R$ : the laser absorption coefficient and the reflectivity of the sample surface; $r_{0}$ : the laser beam radius at $1 / \mathrm{e}$ intensity, $I(t)$ and $I_{0}$ : the intensity of the laser beam and its amplitude; $f, t$ and $i$ : the repetitive rate frequency of the laser, the time and the complex unity; $\xi$ and $\tau$ : the variables of the integration; $\Phi_{L P}$ : the phase of the laser power.

The phase shift between the laser and the thermal power can be found by the expression:

$$
\Delta \varphi=\operatorname{atan}(\operatorname{Re}(\Delta T) / \operatorname{Im}(\Delta T)) .
$$

\subsection{Environmental effect}

The heat exchange with the environment by convection mechanism can be introduced by the conditions of limits at $z=0$ and $z=L[4]$ :

$$
\left.\frac{\partial \Delta T}{\partial z}\right|_{z=0}=\mu\left(T_{z=0}-T_{a}\right) ;\left.\frac{\partial \Delta T}{\partial z}\right|_{z=L}=\mu\left(T_{z=L}-T_{a}\right),
$$

where: $\mu=h / k, h\left(\mathrm{Wm}^{-2} \mathrm{~K}^{-1}\right)$ is a coefficient of thermal exchange with environment by convection. The environment temperature $T_{a}$ is supposed to be equal to the initial temperature of the plate, thus: $T_{z=0}-T_{a}=\Delta T(t, z=0, r)$; $T_{z=L}-T_{a}=\Delta T(t, z=L, r)$. The losses by thermal emission are supposed to be negligible.

The solution of the heat equation:

$$
\begin{aligned}
\rho c \frac{\partial \Delta T}{\partial t}= & k\left(\frac{\partial^{2} \Delta T}{\partial z^{2}}+\frac{\partial^{2} \Delta T}{\partial r^{2}}+\frac{1}{r} \frac{\partial \Delta T}{\partial r}\right) \\
& +\alpha\left(1-R_{c}\right) I(t, r) e^{-\alpha z},
\end{aligned}
$$

on $0 \leq z \leq L$ with the initial condition $\Delta T(t=0, z, r)=0$ was obtained for the $n$th harmonic of the laser repetition frequency:

$$
\begin{aligned}
& \Delta T_{n}(z=0, r)=\frac{\alpha(1-R) \tilde{I}_{n}}{k} \int_{0}^{\infty} \frac{\Theta(\xi)}{\xi^{2}-\alpha^{2}-2 \pi \times i \times n \times f \times \frac{\rho c}{k}} \times J_{0}(\xi r) \\
& \times\left(\frac{e^{-\psi L}-e^{-\alpha L}}{e^{\Psi L}-e^{-\psi L}}\left(\frac{\mu+\alpha}{\mu-\Psi}-\frac{\mu+\alpha}{\mu+\Psi}\right)-\frac{\mu+\alpha}{\mu+\Psi}+1\right) d \xi,
\end{aligned}
$$

where: $\Psi=\sqrt{\xi^{2}-2 \pi \times i \times n \times f \times \rho c / k} ; \mu=h / k$;

$$
\begin{aligned}
& \Theta(\xi)=\left\{\begin{array}{l}
\frac{\xi r_{0}^{2}}{2} \exp \left(-\frac{\xi^{2} r_{0}^{2}}{4}\right) \text { - the Gaussian beam; } \\
r_{0} J_{1}\left(\xi r_{0}\right)-\text { top-hat beam; }
\end{array}\right. \\
& J_{n}(\xi r)=\frac{1}{\pi} \int_{0}^{\pi} \cos (n \tau-\xi r \sin \tau) d \tau ; \tilde{I}_{n}=i \sqrt{a_{n}^{2}+b_{n}^{2}} e^{-i \varphi_{L}^{n}} ; \\
& \varphi_{L}^{n}=a \tan \left(b_{n} / a_{n}\right) ; a_{n}=2 f \int_{0}^{1 / f} I(t) \sin (2 \pi n f t) d t ; \\
& b_{n}=2 f \int_{0}^{1 / f} I(t) \cos (2 \pi n f t) d t .
\end{aligned}
$$



Fig. 2. Phase shift for SS 304L plate of $400 \mu \mathrm{m}$ thickness.

The phase shift can be written as:

$$
\Delta \varphi=\operatorname{atan}\left(\operatorname{Re}\left(\Delta T_{n}\right) / \operatorname{Im}\left(\Delta T_{n}\right)\right) .
$$

It depends on the parameters $k, \rho c, \alpha, L, r_{0}, h$. For thin metal plates, the environmental effect on the phase shift may be considered as negligible. For example, for SS 304L of $400 \mu \mathrm{m}$ thickness, the phase shifts are not affected by the environment even for a material with $h=100 \mathrm{~W} / \mathrm{m}^{2} \mathrm{~K}$ (water) [4].

\subsection{Simple analytical expressions}

The numerical simulation of laser heating is used to fit the calculated phase shifts with the experimental ones by adjusting the material properties. A typical dependence of a phase shift on a laser modulation frequency is presented in Figure 2. For the laser beam with a diameter satisfying $r_{0} / 100 \leq L \leq r_{0} / 2$, one may observe a minimum on the phase shift curve with the corresponding values $\varphi_{\min }$ and $f_{\min }$.

Multiparameter simulation of laser heating enables one to determine the effect of the interaction parameters and the material properties on the phase shifts [3-7]. Two analytical expressions were derived to relate laser parameters, sample properties, $\varphi_{\min }$ and $f_{\min }$. This inter-relationship may provide rapid measurements of thickness $L$ and diffusivity $D$ of a sample with $99 \%$ accuracy:

$$
L[\mu \mathrm{m}]=\left(r_{0}[\mu \mathrm{m}] / \zeta_{\varphi}\right) \times \ln \left(90^{\circ} / \varphi_{\min }\right),
$$

$D\left[\mu \mathrm{m}^{2} / s\right]=\left(1 / \zeta_{f}\right) \times r_{0}[\mu \mathrm{m}] \times L[\mu \mathrm{m}] \times f_{\min }[\mathrm{Hz}]$,

with $\zeta_{\varphi}$ and $\zeta_{f}$ calculated values (Fig. 3).

\section{Experimental}

The experimental setup with a compact fiber laser (low divergent near the Gaussian beam with $\mathrm{M}^{2}=1.1$, beam radius $r_{0} \cong 1 \mathrm{~mm}, 1060 \mathrm{~nm}$ wavelength) is simple in its 

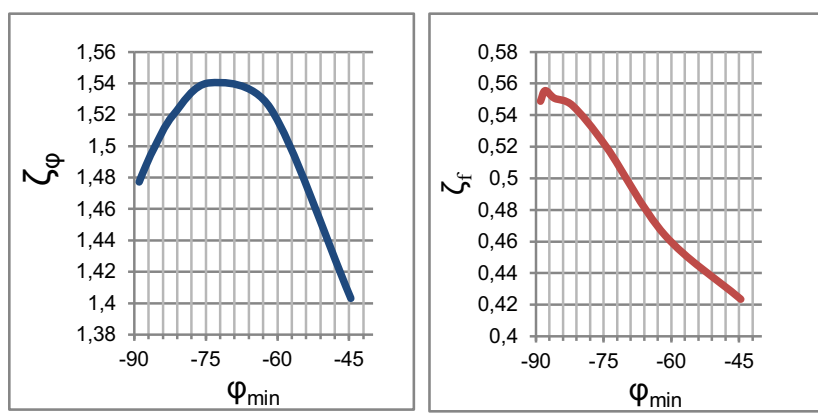

Fig. 3. Calculated values of $\zeta_{\varphi}$ and $\zeta_{f}$ parameters.

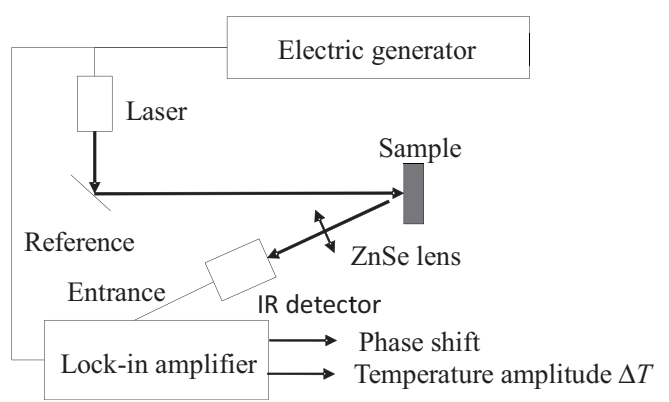

Fig. 4. Scheme of the experimental setup.

arrangement (Fig. 4) and enables one to make remote measurements with a millimeter lateral resolution in a wide distance range (from some centimeters up to several meters).

\subsection{Metal plates characterization}

The lock-in thermography for a plate characterization was validated with a set of etalon samples. The obtained results on plate thickness and diffusivity measurements are presented in reference [3]. These results have demonstrated $\approx 90 \%$ accuracy of thickness and thermal diffusivity measurements. The measured thermal diffusivities along with the referenced values from literature are summarized in Figure 5.

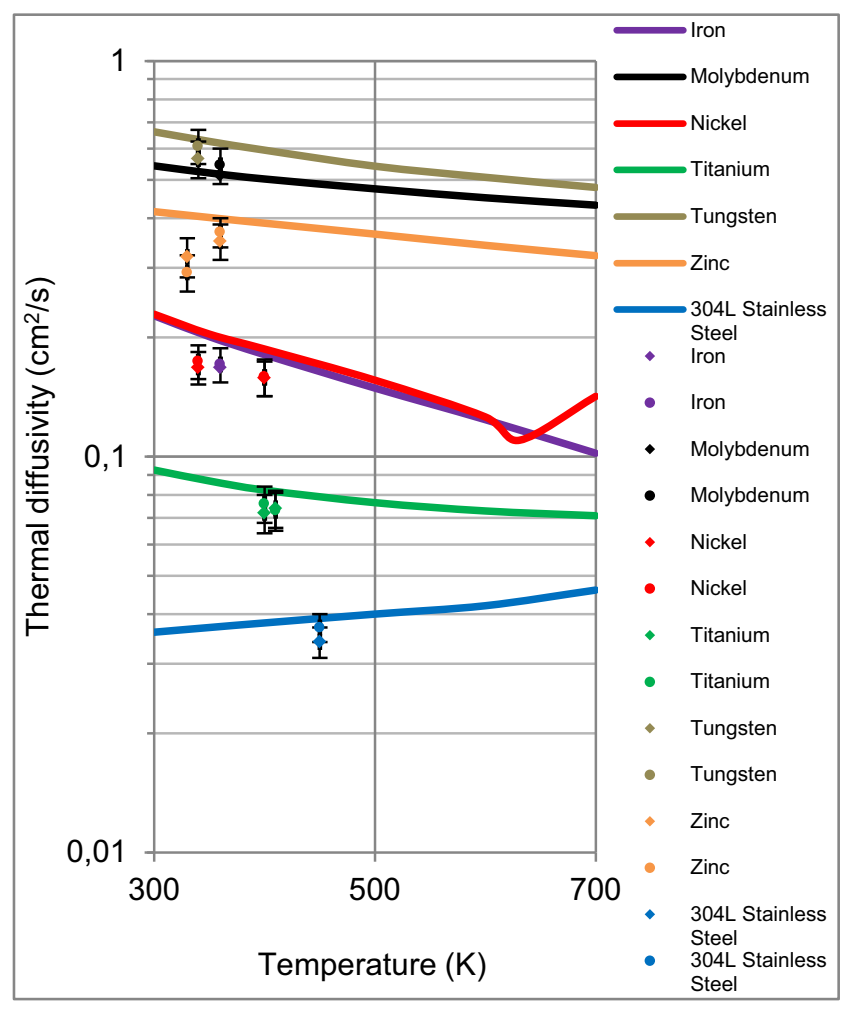

Fig. 5. Comparison of the measured thermal diffusivities along with the referenced values from literature (presented as solid lines) [8]. The square points $-D_{\mathrm{m} 2}$ and the circle points $-D_{\mathrm{m} 1}$ (see Tab. 1 for $D_{\mathrm{m}}$ definition).

\subsection{Zircaloy-4 cladding characterization}

After the characterization of the metal plates by the above procedure, the method was used for studying Zircaloy-4 claddings (Fig. 6). The schematic comparison of the cladding diameter and the one of the heated zone (with the tested zone on it) is presented in Figure 6 . As the heated zone diameter is smaller than the one of $\mathrm{Zy} 4$ cladding, the heated zone may be considered as a plane surface, and thus, the same procedure as the one for metal plates may be followed.

Table 1. Thickness and thermal diffusivity measurements for $\mathrm{Zy} 4$ claddings with oxide layers.

\begin{tabular}{llccc}
\hline \multicolumn{4}{c}{ Zircaloy-4 claddings with oxide layers } \\
\hline Oxide thickness & $\mu \mathrm{m}$ & 5 & 10 & 15 \\
Laser mean power & $\mathrm{W}$ & 2.7 & 2.7 & 2.7 \\
Temperature & ${ }^{\circ}$ & $100 \pm 5$ & $100 \pm 5$ & $100 \pm 5$ \\
$D_{\text {ref }}$ reference & $\mathrm{cm}^{2} / \mathrm{s}$ & $0.073 \pm 0.003$ & $0.073 \pm 0.003$ & $0.073 \pm 0.003$ \\
$L_{\text {ref }}$ reference & $\mu \mathrm{m}$ & $570 \pm 2.5$ & $570 \pm 2.5$ & $570 \pm 2.5$ \\
$r_{0}$ measured & $\mu \mathrm{m}$ & $1740 \pm 30$ & $1740 \pm 30$ & $1740 \pm 30$ \\
$\varphi_{\text {min }}$ measured & $\circ$ & $-53.3 \pm 0.5$ & $-53.1 \pm 0.5$ & $-54.7 \pm 0.5$ \\
$f_{\text {min }}$ measured & $\mathrm{Hz}$ & $3.1 \pm 0.3$ & $3.1 \pm 0.3$ & $3.4 \pm 0.3$ \\
$L_{\mathrm{m}}$ measured & $\mu \mathrm{m}$ & $594 \pm 15$ & $598 \pm 15$ & $564 \pm 15$ \\
$D_{\mathrm{m} 1}$ measured with $L_{\mathrm{m}}$ & $\mathrm{cm}^{2} / \mathrm{s}$ & $0.068 \pm 0.006$ & $0.069 \pm 0.006$ & $0.071 \pm 0.006$ \\
$D_{\mathrm{m} 2}$ measured with $L_{\mathrm{ref}}$ & $\mathrm{cm}^{2} / \mathrm{s}$ & $0.066 \pm 0.006$ & $0.066 \pm 0.006$ & $0.071 \pm 0.006$ \\
\hline
\end{tabular}



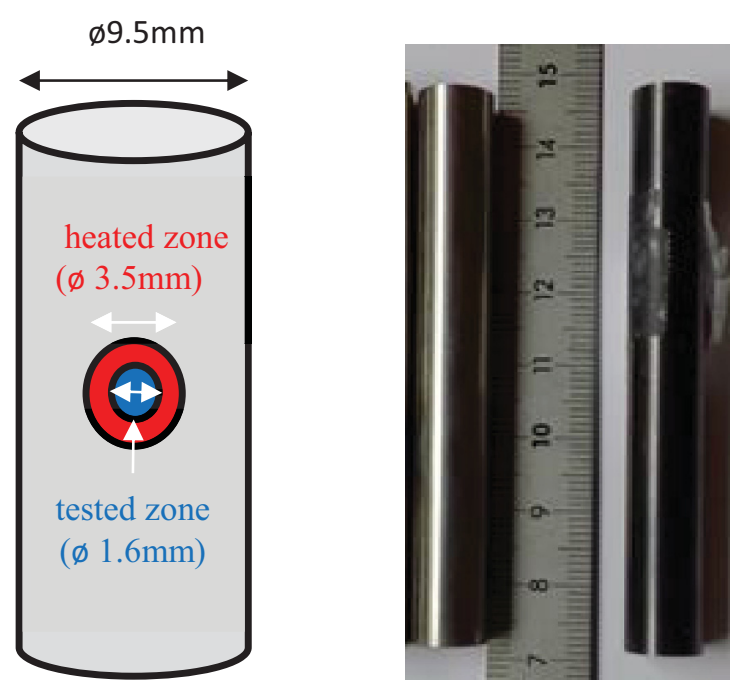

Fig. 6. On the left: schematic comparison of the Zy4 cladding diameter and those of the heated zone (in red) and of the tested zone on it (in blue). On the right: the picture of Zy4 claddings. Some Zy4 claddings were artificially oxidized (on the right in the picture).

To study the effect of the oxide layer on the measured thermal diffusivity, some Zy4 claddings were artificially oxidized in a furnace at different regimes (temperature, environment, time) to obtain oxide layers of different thickness $(5-15 \mu \mathrm{m})$. For $5 \mu \mathrm{m}, 10 \mu \mathrm{m}$, and $15 \mu \mathrm{m}$ oxide layer thickness, the regimes, respectively, were as follows: $\left(500{ }^{\circ} \mathrm{C}\right.$, in air, for 37 hours $),\left(550^{\circ} \mathrm{C}\right.$, in air, for 23 hours $)$, and $\left(550^{\circ} \mathrm{C}\right.$, in water vapor, for 51 hours). The surface of Zy4 with the oxide layer of $10 \mu \mathrm{m}$ thickness has suffered nitriding effect, while the samples with $5 \mu \mathrm{m}$ and $15 \mu \mathrm{m}$ oxides were of a good quality.

The results on Zy4 claddings characterization are presented in Figures 7 and 8 and in Table 1. At low modulation frequency $(f<20 \mathrm{~Hz})$, the phase shift is poorly affected by the presence of the oxide layer (Fig. 7). Thus, for Zircaloy- 4 claddings, $\varphi_{\min }$ and $f_{\min }$ method may be used to determine thickness and thermal diffusivity (see Tab. 1). The measurement relative deviations were less than $10 \%$.

At higher modulation frequency $(f>20 \mathrm{~Hz})$, a clear effect of the oxide layer on the phase shifts is observed (Fig. 7). Due to the fact that $\mathrm{ZrO}_{2}$ layers are semitransparent, the theoretical models for phase shift calculation $[1,2]$ are not applicable in this case. However, there are all the reasons to suppose that further development of the thermal model of heating a semi-transparent layer on a metal plate will provide an adequate on-line in situ characterization of oxide formation.

\section{Conclusions}

The homemade thermal model of the local heating of a homogeneous and isotropic plate with infinite dimensions was developed and verified by characterizing different metal plates and Zircaloy-4 claddings. Two analytical expressions (7) and (8) for sample thickness $L$ and thermal

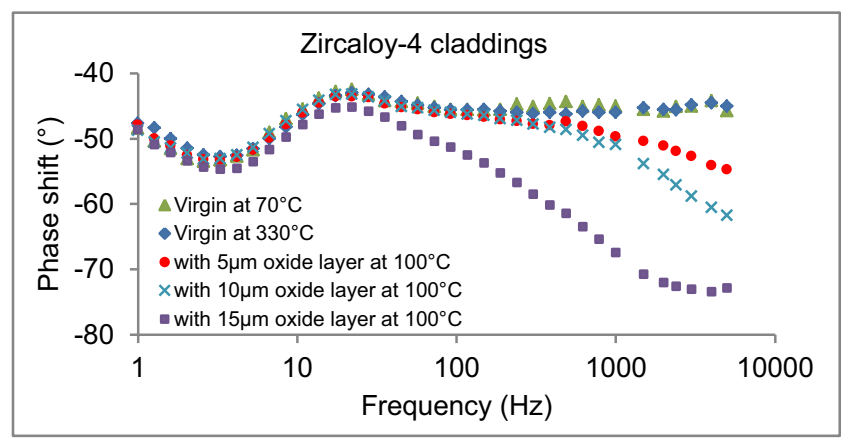

Fig. 7. Phase shifts for Zy4 claddings as a function of modulated frequency.

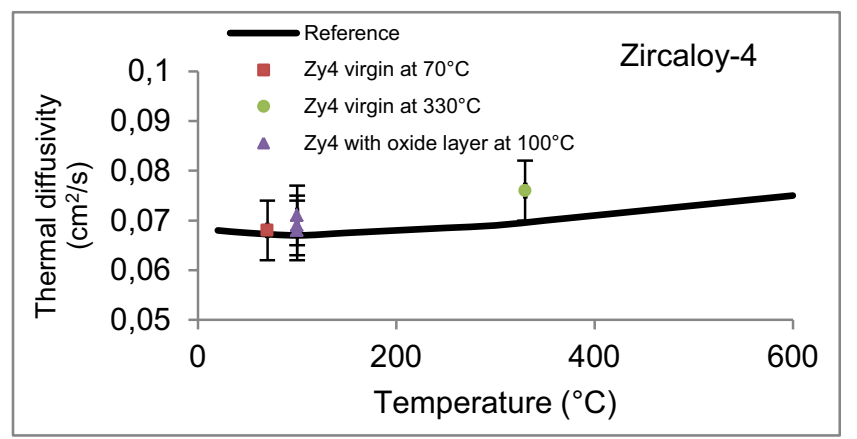

Fig. 8. Measured and reference values of thermal diffusivity as a function of temperature $\left({ }^{\circ} \mathrm{C}\right)$ for $\mathrm{Zy} 4$ claddings.

diffusivity $D$ were derived. These expressions and the lockin thermography measurements (the minimal phase shift $\varphi_{\min }$ and the corresponding minimal modulated frequency $\left.f_{\min }[6,7]\right)$ are used to measure the thickness and the thermal diffusivity of the samples. The obtained results are in agreement with experimental data within an accuracy of $90 \%$. The developed method may be applied for any material with a high absorption coefficient $\alpha$, that is, for any plate with $\alpha L>>1$. Based on the results obtained, we may conclude that a rapid remote in situ control over components in nuclear installations may be ensured with a good spatial resolution (of the order of a laser beam diameter $2 r_{0}$ ).

Authors acknowledge DEN/DANS/DMN/SRMA/LC2M team for Zircaloy-4 claddings supply.

\section{References}

1. A. Semerok, F. Jaubert, S.V. Fomichev et al., Laser lock-in thermography for thermal contact characterisation of surface layer, Nucl. Instrum. Methods Phys. Res. A 693, 98 (2012)

2. A. Semerok, S.V. Fomichev, F. Jaubert et al., Active laser pyrometry and lock-in thermography for characterisation of deposited layer on TEXTOR graphite tile, Nucl. Instrum. Methods Phys. Res. A 738, 25 (2014)

3. S. Pham Tu Quoc, G. Cheymol, A. Semerok, New contactless method for thermal diffusivity measurements using modulated photothermal radiometry, Rev. Sci. Instrum. 85, 054903 (2014) 
4. S. Pham Tu Quoc, Caractérisation des propriétés d'un matériau par radiométrie photothermique modulée, CEA PhD Thesis, France, 2014

5. D. Melyukov, Étude et développement d'une méthode de caractérisation in-situ et à distance de dépôts en couches minces par pyrométrie active laser, CEA PhD Thesis, France, 2011
6. D. Melyukov, P.-Y. Thro, Patent CEA FR2980846-A1, Procédé de détermination sans contact de l'épaisseur d'un échantillon, 2013

7. S. Pham Tu Quoc, G. Cheymol, A. Semerok, Patent CEA FR1355905, Procédé de détermination de la diffusivité thermique et système pour la mise en œuvre, 2013

8. Y.S. Touloukian, R.W. Powell, C.Y. Ho, M.C. Nicolaou, Thermal diffusivity (IFI/Plenum, New York, 1973)

Cite this article as: Alexandre Semerok, Sang Pham Tu Quoc, Guy Cheymol, Catherine Gallou, Hicham Maskrot, Gilles Moutiers, Lock-in thermography for characterization of nuclear materials, EPJ Nuclear Sci. Technol. 2, 20 (2016) 